\title{
Metastasis of Cervical Cancer to the Abdominal Wall: a case report
}

\author{
Khalife D. ${ }^{2}$, Husheimi A. ${ }^{2}$, Abiad M. ${ }^{3}$, Rammal R. ${ }^{4}$, Abdallah R. ${ }^{1}$
}

1. Division of Gynecologic Oncology, Department of Obstetrics and Gynecology, American University of Beirut Medical Center, Lebanon

2. Department of Obstetrics and Gynecology, American University of Beirut Medical Center, Lebanon

3. Faculty of Medicine, American University of Beirut, Lebanon

4. Department of Pathology \& laboratory medicine, American University of Beirut Medical Center, Lebanon

\section{Introduction}

Squamous cell carcinoma of the cervix (SCC) accounts for $85 \%$ of the cases of cervical cancer.

The most common sites of recurrence postoperatively are in the pelvis and vagina. Metastases to the abdominal wall from cervical carcinoma are rare with a reported incidence of 0.1 to $2 \%$, and a survival rate ranging from 1 week to 7 months.

\section{Case report}

A 43-year-old woman with stage IB2 SCC of the cervix underwent a radical hysterectomy, with bilateral salpingo-oophorectomy and pelvic lymph node dissection at a different institution. She did not receive any adjuvant treatment, although she is considered at intermediate risk by Sedlis criteria (size of $4 \mathrm{~cm}$ and deep stromal invasion). Fifteen months later, she presented to our institution with a painful suprapubic rapidly growing mass. MRI of pelvis showed a $13 \times 11 \times 9 \mathrm{~cm}$ mass adherent to the anterior abdominal wall, to the dome of the bladder and to the small bowel with enlarged right pelvic lymph nodes. There was no evidence of intra-abdominal or distant metastases. The mass was removed en bloc, with rectus abdominus muscles, 2 segments of small bowel and a portion of the bladder, along with right pelvic lymphadenectomy. The resultant infra-umbilical abdominal wall defect measured $20 \times 15 \mathrm{~cm}$. It was reconstructed with an island pedicle of right thigh anterolateral vastus and vastus lateralis muscle flap. Histology showed invasive non-keratinizing moderately differentiated SCC, consistent with uterine cervical primary, involving urinary bladder, bowel and soft tissue, with negative margins and one positive right pelvic lymph node.
Adjuvant treatment was delayed for 3 months to allow for complete healing as the patient had to undergo deepithelialization of a segment of the flap. She received 6 cycles of CarboplatinPaclitaxel and Bevacizumab, and was then lost to follow up for 4 months. CT scan confirmed the absence of recurrence and she is currently receiving the pre-planned radiation therapy. She remains free of recurrence after 12 months of the surgery.

\section{Discussion}

We describe the case of a 43-year-old woman with abdominal wall lesion that turned out to be metastatic from primary cervical carcinoma. Metastatic carcinoma to the abdominal wall is uncommon. Treatment involves chemotherapy and/or radiotherapy. Two cases in the literature are similar to our case, and treatment consisted of palliative resection of the abdominal wall lesion, followed by reconstruction with various muscular flaps. Recurrence is deemed to be the result of residual occult cancer after surgical resection. Other mechanisms of spread proposed are through implantation of cancer cells at the time of surgery or via retrograde spread through the lymphatics.

\section{Conclusion}

The present case is uncommon because abdominal wall metastasis from SCC of the cervix after radical surgery is rare. It was also challenging since the removal of the lesion has resulted in a large soft tissue defect. However, with advancement in reconstructive surgery, extensive resection with defect closure with rotational flaps may be the standard of care in the management of abdominal wall lesion after cervical cancer in order to improve the overall survival.

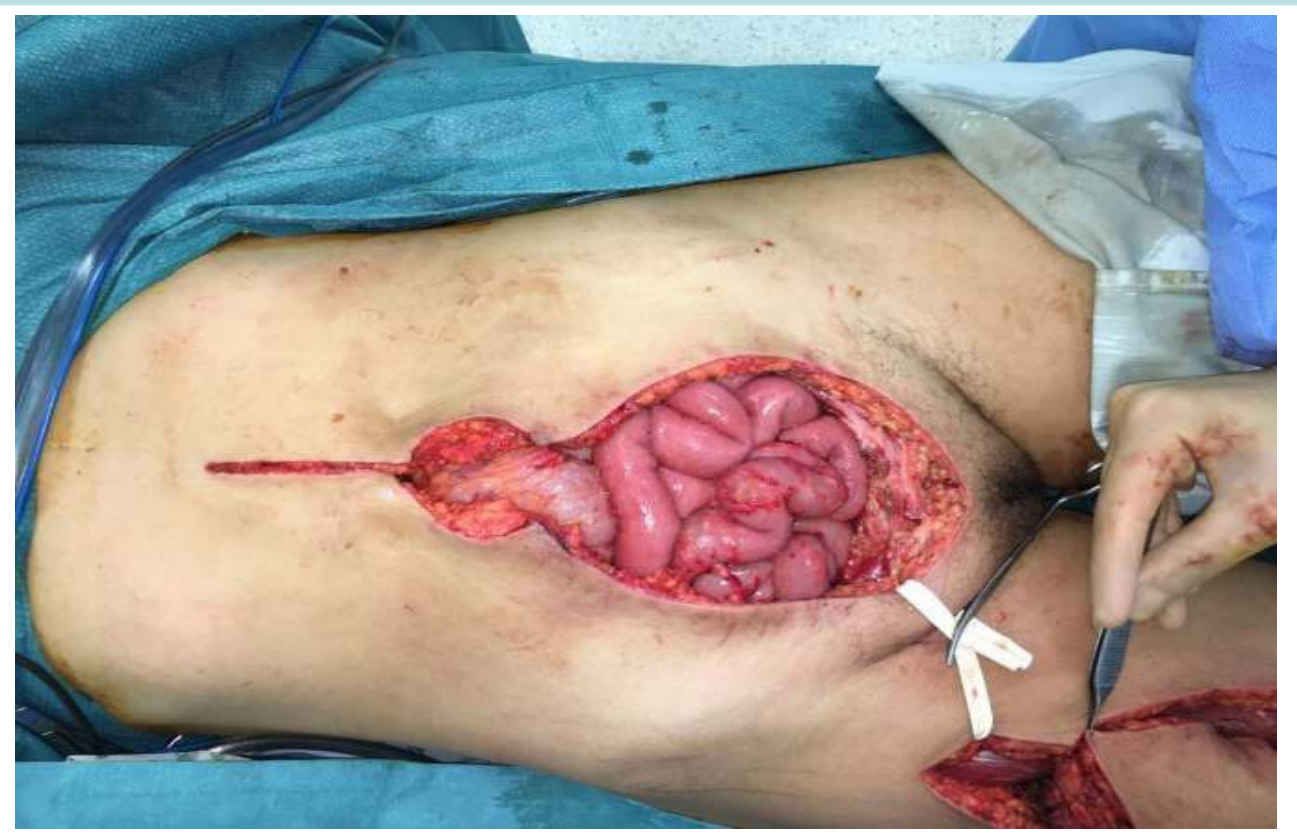

Boos, A. M., Beckmann, M. W., Horch, R. E., \& Beier, J. P. (2014). Interdisciplinary treatment for cutaneous abdominal wall metastasis from cervical cancer with resection and reconstruction of the abdominal wall using free latissimus dorsi muscle flap: a case report. Geburtshilfe und Frauenheilkunde, $74(6), 574$. 